\title{
Remote Controlled Security Door
}

\section{Ekejiuba $\mathrm{CO}$ and Folayan GB*}

Department of Electrical/Electronic Engineering, The Federal Polytechnic Ado-Ekiti, Nigeria

\begin{abstract}
The ultimate purpose of this paper is to develop a remote controlled security door using a microcontroller. It is a means of enforcing security features of the entire building structure, equipped with appropriate management control. In this paper we have designed system that allows unlocking a secure door remotely. The main aim of this paper is to help users improve the door security of sensitive locations, from car parks, shopping centres or corporate head offices to airports, banks or nuclear power plants. This paper takes a cursory look at other security methods of controlling access, their disadvantages and then goes on to the design of the hardware as well as software programming to lock or unlock the door. Both software and hardware for the system were developed in this paper by using microcontrollers, relays, sensors, induction motors and some mechanical system to demonstrate the response of the output and input devices.
\end{abstract}

Keywords: Hardware; Software; Remote control; Microcontroller; Security door

\section{Introduction}

Due to the need to increase the security of life and properties in our environment, security systems are used as selective systems to decide who have access to a given location based on a personal distinctive trait of the user. It also serves the function of sensing or detecting false intrusion (using input sensory devices and gives early warning using audio warning devices alarm - light indicators and remotely controlled computer systems etc). The term false intrusion here is used to mean any form of attempt to gain entry without following the proper predesigned protocol(s). From time past until present, security locks usually includes mechanical devices made of forged metal i.e. simple lock and bolt, the door chain, pin tumbler lock, the jam lock and padlock etc. Other recently developed security devices are gadgets like laser beam detectors, motion detectors and magnetic card readers. Most recent of these devices are offshoots of biometric engineering. They include voice recognition systems, finger print readers, retina eye scanners, etc. [1]. The major characteristic of security devices is to prevent an intruder from gaining access to a location. Most of these devices however have lapses which give an unauthorized person access to where they are barred i.e. the simple jam lock and pad lock can be forced open or the keys duplicated by unauthorized persons and under certain conditions, i.e. physical changes of the individual concerned, the biometric devices sometimes fail in recognizing the authentic individual concerned [2]. Hence they cannot be totally relied on. Furthermore, these devices are very expensive [3], its use is restricted to only a few individuals or organizations that can afford it. Biometric systems may violate users privacy while some users consider them to be personally invasive and may also imply loss of anonymity [2]. Devices such as motion detectors, light detectors among others are susceptible to be triggered by false signals such as noise impulses, whenever its sensitivity is increased [2]. They are not suitable for most outdoor security protection due to the fact that they don't posses high discriminative capability during operation and they are fairly expensive.

It is hence imperative to provide a security door, one which is also efficient and reliable; with high discriminative capability, non reliance on the physical quality of the individual concerned does not have key access or any physical locking interface and which is by far less expensive than their counterparts [4]. This has led to the design and construction a remote control security door using microcontroller. It includes remote control and using correct personal identification numbers to operate the locking device manually, by conditioning the access based on the configuration of the security device thereby granting access to the user with correct pin number.

The remote control security door finds application in homes, banks in the field of military applications i.e. ammunition ware house, industries and ministries and Government Parastatals, airports nuclear power plants etc.

There are three important components of access control: Identification, Authentication, and Authorization. Identification is the activity of the subject supplying information to identify itself to an authentication service. Authentication is the second part of a credential set to verify the identity of the subject. These mechanisms could be passphrases, passwords, cryptographic keys, PIN numbers, or tokens. Authorization is the process of determining what this identified subject can actually access and what operations it can carry out [5].

\section{Methodology}

The modular method is employed in the design. The design involves two parts; the hardware and software parts.

\section{Hardware design}

The hardware part is designed to drive an induction motor for the required application in forward and reverse directions using wireless technology. The proposed system demonstrates a technology to rotate a squirrel cage induction motor in both clockwise and counter clockwise direction. It also has the provision to control the direction of the motor using an IR remote.

When the remote button is pressed, it sends an IR signal in RC5 code which is received by an IR receiver called TSOP-1738. Output from the receiver is fed to a microcontroller of 8051 family which is

*Corresponding author: Folayan GB, Department of Electrical/Electronic Engineering, The Federal Polytechnic Ado-Ekiti, Nigeria, Tel: +2348035971444; E-mail: nattygbengu@gmail.com

Received April 21, 2016; Accepted April 23, 2016; Published May 27, 2016

Citation: Ekejiuba CO, Folayan GB (2016) Remote Controlled Security Door. J Electr Electron Syst 5: 184. doi:10.4172/2332-0796.1000184

Copyright: (c) 2016 Ekejiuba CO, et al. This is an open-access article distributed under the terms of the Creative Commons Attribution License, which permits unrestricted use, distribution, and reproduction in any medium, provided the original author and source are credited. 
interfaced to a relay driver IC. Thereafter, the relay switching is done in by-stable mode for a split-phase induction motor to rotate in forward and reverse directions thereby controlling the Link mechanism to open or close the door Figure 1.

The software is designed in order to support the effectiveness of the hardware device. The complex and intricate operating routine of the software is achieved by writing the program in modules starting with the program algorithm, followed by the program flowchart as shown in the Figure 2. The software was written in Micro basic language, and was written in sections for easy debugging and troubleshooting. Each section is tailored to meet the duty that will be imposed on the corresponding hardware unit.

The microcontroller program was developed that is compiled, burnt and evaluated before it was transformed to the microcontroller through the programmer. The approach is by experimental modular design where each is analyzed extensively and tested for functionality so as to prove satisfactory before joining the blocks to form the overall circuit [6].

The modules involved in this project are:

a. The power supply module (DC $12 \mathrm{~V})$

b. The keypad unit

c. The display unit

d. The Induction Motor

e. The control unit (microcontroller PIC16F877A)

f. The Link Mechanism.

\section{Keyless entry system}

A remote keyless system is a system designed to remotely permit or deny access to premises or automobiles. There are several remote keyless entry (RKE) systems on the market, including but not limited to Kee Loq by Microchip, HITAG by Philips, and AVR411 by Atmel.

The oldest known mechanical functioning lock was an Egyptian door lock used about 2000 BC, made of wood and fastened vertically on the door post, the wooden block contained moveable pins or "pin tumblers" that dropped by gravity into openings in the cross piece of "bolts" and locked the door. The major disadvantage with it is that it was wholly made of wood. The Romans made an improvement on this by fabricating the first metal locks which was later improved by Robert

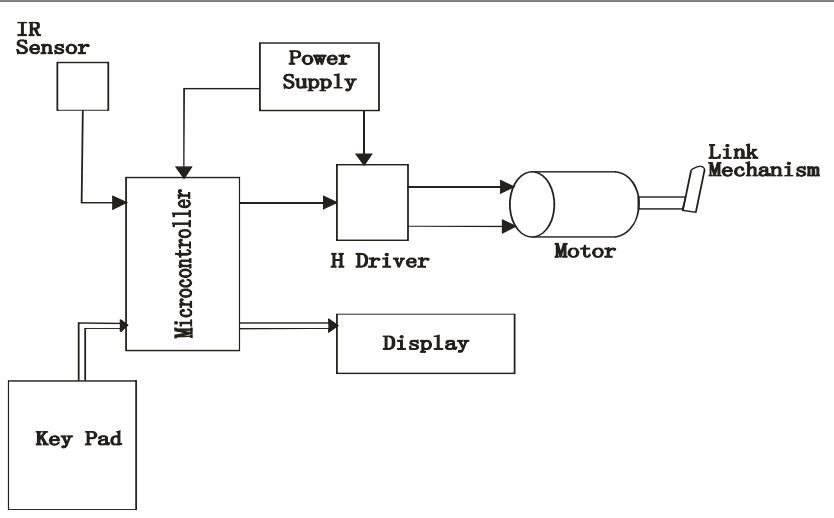

Figure 1: Block diagram of a microcontroller based remote control security door.

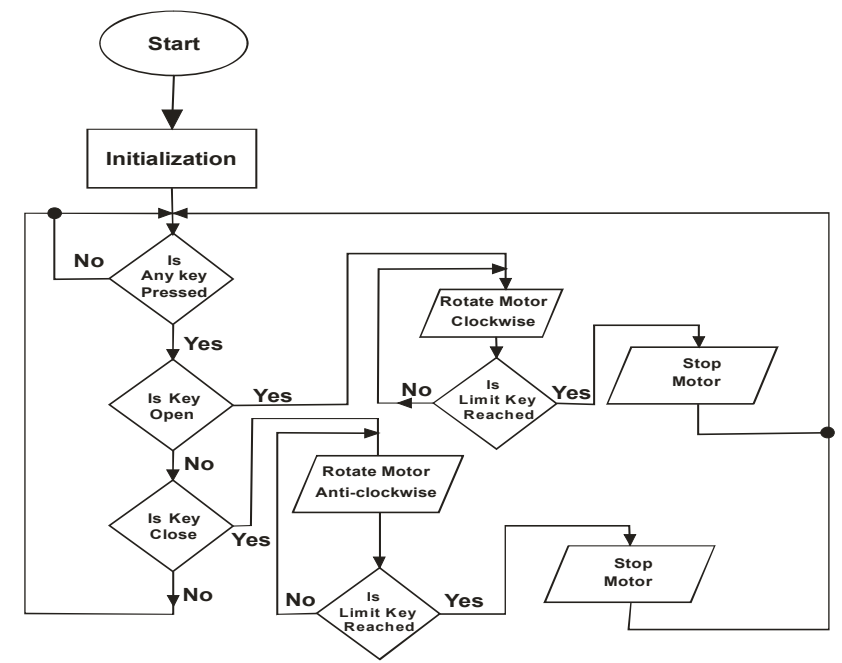

Figure 2: System flow chart.

Barson, an English man in 1778 and Linus Yale Jnr. an American in 1861 [7]. In the late 20th century, electromechanical locks were developed to trip electrical circuit as seen in automobile ignitions. Other keyless locks include remote controlled lock, "security card" operated and electronic code lock. This report is about remote control security door, which is designed to respond to an electronic logic signal mechanism, with a digit sequence counter performing the function of a key. They are operated by inputting the correct code by external means through a 5 keypad into a microcontroller which already have a pin number in its EEPROM memory (internal) to compare with, so that if it is correct (that is the pin number inputted by the user), It activates the port to which the induction motor is connected (the load that operate the door).

\section{The control unit}

The microcontroller PIC16F877A is the main control unit. PIC16F877A microcontroller was picked for several reasons. Firstly, it is operated on a $+5 \mathrm{~V}$ DC supply and draws very little current. Furthermore, it has a very low power dissipation and high speed of operation and still maintains its data in case of power loss. Finally it has a large storage memory. It processes and verifies the keypad inputs (Entered pin), and generate control signals to power the Induction motor attached to the Link mechanism. Thus, this is done by comparing the digits with the right PIN number in the EEPROM memory (internal), if it is correct, it activates the port RD1which is connected to the Induction motor (this represents the load), and also send an output text display on the LCD, indicating "Enable mode [8].

\section{The keypad unit}

The keypad is employed as an external interface to the system through, the output of the button pressed on the keypad is displayed on the LCD, this help to makes correction if a mistake is made during the typing of the codes. When the input pin is correct it causes the microcontroller to generate the control to drive the Induction Motor either to open or close the door [9].

\section{The display panel unit}

The display used is the $16 \times 2 \mathrm{LCD}$ liquid crystal display so as to give the user the opportunity of textual display as compared to conventional ones which shows digits. $16 \times 2$ LCD uses a serial protocol having 8 
pins with 4 pins used for communication with the microcontroller and the rest four pins used for other various function such as Reset, Ground, Vout and Vdd of 5 V to run the LCD.

\section{Software design.}

The software is designed in order to support the effectiveness of the hardware device. The complex and intricate operating routine of the software is achieved by writing the program in modules starting with the program algorithm, followed by the program flowchart as shown in the Figure 2. The software was written in Mikrobasic language, and was written in sections for easy debugging and troubleshooting. Each section is tailored to meet the duty that will be imposed on the corresponding hardware unit.

\section{Display program}

The Display panel unit display up to twelve different statutes based on the operating state of the device, these statutes are "OPEN", "ENTER PIN", "Enable Mode", "Disable Mode", "ENTER OLD PIN", "ENTER NEW PIN", "PIN MISMATCHED", "PIN CHANGED", "ENTER NEW PIN AGAIN", "HIDE OR DISPLAY NUMBER", "PRESS ANY KEY", "CLOSE DEVICE". The design of the font was done using the

LCD font designer in the mikrobasic crack.

\section{Keypad program}

Apart from the programming of the key pad to coordinating the activities of the microcontroller, another major programming done here is preventing jumping of the input key (bouncing). The program scans each key to detect a depressed key, and then a de-bouncing routine which is about $150 \mathrm{~ms}$ delay is then executed. The routine is important so as to decipher authentic key pressed from Electrical noise which can lead to multiple input. The program decodes an authentic key pressed and stores the numeric values in a given register, it repeats this until the required numbers of key inputs are collected are collected. The "Ok" function key then enters the values to be processed.

\section{Relevance of remote controlled using infrared}

1) Ability to interface with infra-red device

2) Create a user-friendly web interface to access and operate door strike

3) Provides a feedback log of date and times in which the door was operated.

\section{Operation of remote controlled security door}

The voltage is supplied from the relay is supplied from the supply authority to the relay 1 " that is used as a priority. One of the coil terminals of the relay 1 was connected to a transistor. That is normally closed terminal through its common terminal. The coil is energized at the present time, the N.C contact will open then, the contacts of the relay 1 are made to produce the head (output) during this process relay 2 is energized being that, the coil or relay 2 was connected to N.O terminal through its common terminal.

When the coil is energized by the battery the contacts of relay 2 are made and heard is supplies then relay 1 de-energized, we choose to powered the device by a battery since its easier from the PIC in combination with a relay, in addition to the door itself, there is an infra-red receiver and a check module. $38 \mathrm{khz}$ signals are outputted by the serial infra-red control unit (SIRCU) it consist of a sensor and limited amount of logic. The pic samples the output every $1.365 \mathrm{~ms}$ which 5 times faster than the sent frequency.

The IR receiver is another piece of hardware situated near the door. If a status button is selected a new window displays, the status of the door. It will either show locked or unlocked or error or an invalid signal sent from the sender unit.

\section{Results}

\section{Testing and performance}

The following tests are carried out on the project:

a) Continuity Test

b) Insulation resistance test

c) Earth leakage test

d) Performances test

a) Continuity test: This test was carried out to ascertain whether there is no open circuit between the contacts. According to the $16^{\text {th }}$ edition of IEE regulation, the continuity test must be carried out to known whether there is open circuit or not in the relay. The testing result display zero in the multimeter [10].

b) Insulation resistance: This is carried out to determine whether there is no breakdown of insulation in the coil of the relay. The testing result display, infinity in the multimeter.

c) Earth leakage: This is to determine whether there is no linkage between the contacts of the relay to the ground. The testing result display, zero in the multimeter.

d) Performance test: The project is Remote Controlled security door System". Its performance range of operation and distance coverage of operation. If the battery is fully charged it can work for at least $20 \mathrm{HR}$, the battery is $12 \mathrm{v}, 7.2 \mathrm{Ah}$ and secondly distance coverage is $9.4 \mathrm{~m}$ long, but 30 feet in meters conversion, the rate at which the door opens and closes is $5 \mathrm{sec}$ (seconds)

After construction of the entire system, the hardware parts including the

Power supply, the $162 \mathrm{LCD}$, the keypad were tested on the bread board while the software debugging was done using the micro basic crack simulator

\section{Testing of the power supply unit}

The power supply circuit was connected on the breadboard with voltmeters connected across some points to get the output voltages and currents.

\section{Key pad unit testing}

Keypad is tested for continuity by keypad is tested connecting an avometer across each button

The $16 \times 2$ LCD was tested by connecting the variable resistor between the Vout and the $\mathrm{V}_{\mathrm{dd}}$ legs of the $16 \times 2 \mathrm{LCD}$.

\section{Discussion}

It is economically wise to use Remote controlled security door using relay since most remote controlled door uses card reader, this project will be of great importance because two relays were used for the supply and two transistors that powered the relays instead of costly circuit. 


\section{Efficiency of the project}

The project is designed to operate specifically at $230 \mathrm{v}$ A.C, and DC $12 \mathrm{~V}$ battery of $7.2 \mathrm{Ah} / 20 \mathrm{HR}$ efficiency without or with little loss. The range or distance of operation of this project is $9.4 \mathrm{~m}$ long.

\section{Summary, Conclusion and Recommendation}

\section{Summary}

The design and construction of remote controlled security door capable of delivering $+230 \mathrm{~V}$ A.C from a $12 \mathrm{D} \mathrm{C}$ input, for as a long as D.C supply is available has been achieved. The major stages are the programmed circuit, supply stage, microcontroller stage and relay stage. Two relay were also used and two transistor which is used to power the relay so that in operating system to locked the door the polarity of the relay and transistor changes. The battery used to power the device is $12 \mathrm{~V}, 7.2 \mathrm{AH} / 20 \mathrm{HR}$, and distance of operation of project is $9.4 \mathrm{~m}$ long.

\section{Conclusion}

The security remote door is capable of delivering a $+230 \mathrm{~V} \mathrm{AC}$, $50 \mathrm{HZ}$ supply from an input voltage of $12 \mathrm{~V} \mathrm{DC}$ switching rate of the programmed circuit determines the rate of operation of the output A C signal delivered to the load. The project involves an infra-red communication between an infra-red activated door and our serial infra-red control unit [SIRCU] device and communication between the SIRCU sensor and a PIC located within the house and denies entry to a room or building.

\section{Recommendation}

Even though the design objective was reasonably achieved, I recommended that this project be improved upon in the following areas:
1. The use of security door is to keep safety of rampaging a building or house.

2. Protection of security and checking security situation.

3. Security, remote controlled to protect security of life and properties.

4. Security door should be developed so that the manufacturing industries can employ this alternative means to protect properties from theft.

\section{References}

1. Prabhakar S, Pankanti S, Jain AK (2003) Biometric recognition: security and privacy concerns. IEEE Security \& Privacy Magazine 1: 33-42.

2. Matyás V, Ríha Z (2002) Biometric authentication - security and usability 227 239

3. Chien L (2011) A Survey of Biometrics Security Systems.

4. Alsaadi IM (2015) Physiological biometric authentication systems advantages, disadvantages and future development a review. International journal of scientific \& technology research 4: 285-289.

5. Mahdi SA (2013) Development of Anti-Theft Door System for Security 4: 237242

6. Hageman S (1999) PIC Development on a Shoestring

7. Rodney C (2004) Scientific American Inventions and discoveries. New Jersey: John Wiley \& Sons.

8. Bates M (2011) An Introduction to Microelectronics, programming techniques. PIC Microcontrollers,3rdedn ,London

9. Souza SD (2002) Multiplexing LED drive and a $4 \times 4$ keypad. Microchip Technology Inc.

10. Mason CR (1956) The art and science of protective relaying, Wiley, London. 